\title{
Cerebral salt wasting following traumatic brain injury
}

\author{
Peter Taylor1,2, Sasan Dehbozorgi33, Arshiya Tabasum1, Anna Scholz', Harsh Bhatt3, \\ Philippa Stewart3, Pranav Kumar1, Mohd S Draman1,2, Alastair Watt4, Aled Rees1,5, \\ Caroline Hayhurst ${ }^{3}$ and Stephen Davies ${ }^{1}$
}

1Department of Diabetes and Endocrinology, University Hospital of Wales, Heath Park, Cardiff, UK, 2Thyroid Research Group, Division of Infection and Immunity, School of Medicine, Cardiff University, Heath Park, Cardiff, UK, ${ }^{3}$ Department of Neurosurgery, University Hospital of Wales, Heath Park, Cardiff, UK, ${ }^{4}$ Department of Diabetes and Endocrinology, North Devon District Hospital, Barnstaple, UK, and 5 Institute of Neuroscience and Mental Health Research Institute, School of Medicine, Cardiff University, Cardiff, UK

\author{
Correspondence \\ should be addressed \\ to P Taylor \\ Email \\ taylorpn@cardiff.ac.uk
}

\section{Summary}

Hyponatraemia is the most commonly encountered electrolyte disturbance in neurological high dependency and intensive care units. Cerebral salt wasting (CSW) is the most elusive and challenging of the causes of hyponatraemia, and it is vital to distinguish it from the more familiar syndrome of inappropriate antidiuretic hormone (SIADH). Managing CSW requires correction of the intravascular volume depletion and hyponatraemia, as well as mitigation of on-going substantial sodium losses. Herein we describe a challenging case of CSW requiring large doses of hypertonic saline and the subsequent substantial benefit with the addition of fludrocortisone.

\section{Learning points:}

- The diagnosis of CSW requires a high index of suspicion. Distinguishing it from SIADH is essential to enable prompt treatment in order to prevent severe hyponatraemia.

- The hallmarks of substantial CSW are hyponatraemia, reduced volume status and inappropriately high renal sodium loss.

- Substantial volumes of hypertonic saline may be required for a prolonged period of time to correct volume and sodium deficits.

- Fludrocortisone has a role in the management of CSW. It likely reduces the doses of hypertonic saline required and can maintain serum sodium levels of hypertonic saline.

\section{Background}

Cerebral salt wasting (CSW) is an uncommon cause of hyponatraemia and is particularly challenging to manage. Making the diagnosis rapidly is vital as its treatment requires volume resuscitation and sodium restoration rather than the water restriction used for the more commonly observed SIADH. Hyponatraemia is common in neurosurgical patients and is associated with substantial morbidity and mortality. Diagnosis of the cause and its correction are essential to prevent severe hyponatraemia, which can substantially worsen cerebral oedema and increase the risk of seizures.

\section{Case presentation}

A 44-year-old male with no substantial medical history was injured falling off his road bike at $25 \mathrm{mph}$ and 
sustained a traumatic brain injury resulting in a marked expressive dysphasia. CT brain demonstrated a small left subdural haematoma, left temporal contusion and right petrous temporal bone fracture. He did not require surgical intervention. Forty eight hours after injury he developed a lower motor neurone facial weakness secondary to the temporal bone fracture, therefore dexamethasone was commenced.

On Day 5 of admission his sodium level fell. Initially attributed to SIADH, his sodium continued to fall rapidly despite fluid restriction and slow sodium tablets. Baseline paired serum and urine osmolality were 265 and 595 respectively with urine sodium of $191 \mathrm{mmol} / \mathrm{L}$. He was referred to endocrinology who suspected a diagnosis of CSW on the basis of failure of resolution with fluid restriction, low JVP and high urine output. Urine output assessment was challenging to interpret initially due to extreme confusion, and agitation preventing catheterisation but his urine output was in excess of $3 \mathrm{~L}$ a day at this point despite a $1.5 \mathrm{~L}$ fluid restriction. During this time the patient recalls having excessive thirst and a craving for the salt tablets, which he later found unpalatable.

\section{Investigation}

Despite hypertonic saline $(1.8 \%$ saline $)$ being commenced, his sodium continued to fall, with increased cerebral oedema and worsening confusion. This combination necessitated admission to intensive care with a sodium nadir of $116 \mathrm{mmol} / \mathrm{L}$. A graph of his serum sodium, urine sodium and daily intravenous sodium is shown in Fig. 1.

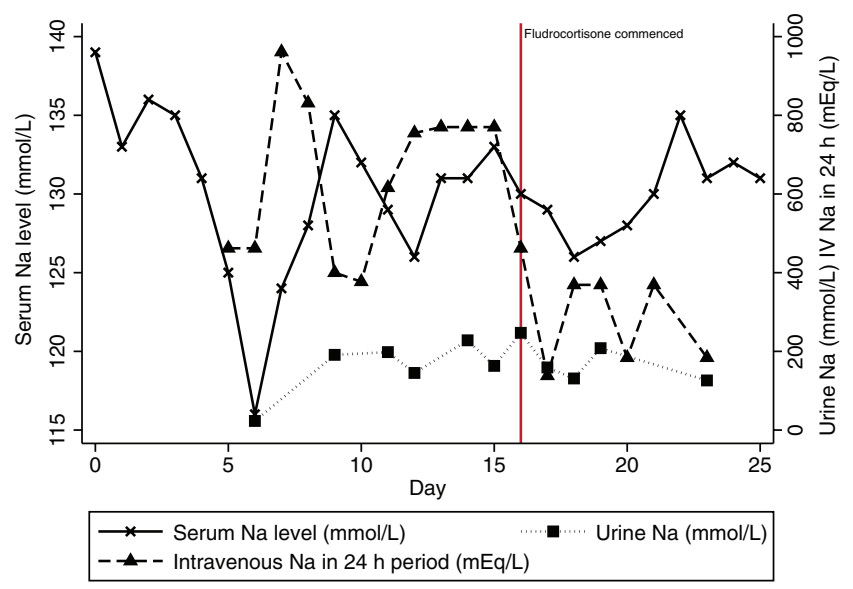

Figure 1

Serum sodium, urine sodium and daily intravenous sodium.

\section{Treatment}

Infusion of $5 \%$ saline ( $>950 \mathrm{mEq}$ of sodium required in first $24 \mathrm{~h}$ in ITU) restored serum sodium to low normal levels within $72 \mathrm{~h}$ (sodium $134 \mathrm{mmol} / \mathrm{L}$ ). During his intensive care admission he also required inotropic support which further highlights the extent of his volume depletion. Following the stabilisation of his sodium levels he was stepped back down to the neurosurgical high dependency unit. However, his urinary sodium remained persistently elevated peaking at $254 \mathrm{mmol} / \mathrm{L}$, (urine osmolality $710 \mathrm{mmol} / \mathrm{kg}$ ) serum sodium fell to $125 \mathrm{mmol} / \mathrm{L}$ despite hypertonic saline and he required substantial volume replacement (equivalent of more than $4 \mathrm{~L} /$ day of $1.8 \%$ saline). Urine output remained persistently high between 3 and $5 \mathrm{~L}$ a day over this time.

Fludrocortisone was therefore introduced at $75 \mu \mathrm{gbd}$ and ultimately increased to $150 \mu \mathrm{gbd}$, which resulted in a substantial (threefold) fall in requirements for hypertonic saline that was still required for another 7 days. His sodium remained stable around $130 \mathrm{mmol} / \mathrm{L}$ on fludrocortisone alone and he was discharged home 25 days post injury. Fludrocortisone was continued for the next four months.

The dexamethasone initiated at 48 hours was continued for a 1-week course. As a baseline cortisol taken $24 \mathrm{~h}$ post the final dexamethasone was $94 \mathrm{nmol} / \mathrm{L}$, he was maintained on hydrocortisone $10 \mathrm{mg}$ bd which was continued until after discharge. A post discharge short synacthen test was normal and hydrocortisone was then stopped.

\section{Outcome and follow-up}

It took four months to wean him off the fludrocortisone without the occurrence of rebound hyponatraemia suggestive of on-going, albeit mild, CSW. Intriguingly, the attempt in the week after he developed peripheral oedema on fludrocortisone was successful and he has remained stable off fludrocortisone for three months. Despite an otherwise excellent recovery, he has since developed hypertension and has observed his sweat appears to be less salty, indicating perhaps on-going mild disturbance of sodium handling.

\section{Discussion}

CSW is a disorder of sodium and water homoeostasis which occurs as a result of cerebral disease in the setting of normal renal function. It was first described over 65 years ago by Peters et al. (1) several years before the SIADH was first described. Unlike SIADH, CSW results in inappropriate 
primary sodium losses which are not related to volume expansion. Despite our awareness of CSW for several decades the pathogenesis remains unclear; however atrial, brain $\mathrm{C}$ type and dendroapsis natriuretic peptides have been implicated $(2,3)$. The release of these peptides may arise from increased atrial stretch and increased ventricular load from sympathetic outflow surges (4) whereas direct trauma to the brain releases BNP. Furthermore ANP and BNP may be released by the hypothalamus as a protective mechanism against rising intracranial pressure (2). Indeed BNP appears to be the driving factor of CSW following sub-arachnoid haemorrhage (2).

It is postulated that these natriuretic peptides inhibit sympathetic outflow, the renin-aldosterone system and the production of vasoconstrictor peptides. This results in a reduction in the efficacy of aldosterone and leads to a substantial reduction in the ability of the kidneys to resorb sodium. Direct interference to the sympathetic innervation to the kidney has also been postulated in the pathogenesis of CSW (5). As a result, there can be substantial impairment of sodium handling.

Whilst CSW should be considered in any at-risk patient with abnormal sodium handling in the absence of renal dysfunction, adrenal insufficiency or inappropriate volume expansion, it is the more severe forms that likely require specialist endocrine input. Severe CSW will likely result in a very high urine sodium concentration, a high urine output leading to a high serum urea, postural hypotension and a low central venous pressure. High fractional excretion of uric acid occurs in both SIADH and CSW; however, this can persist in CSW despite correction of hyponatraemia unlike in SIADH (6).

The management of profound CSW is therefore correction of intravascular volume depletion and hyponatraemia, and mitigation of the on-going high urinary sodium loss. The $\mathrm{Na}$ losses are such that hypertonic saline is usually necessary. Close monitoring of hypertonic saline is essential to prevent overly rapid correction of hyponatraemia, which might result in osmotic myelinolysis. Addressing these issues reinforces the importance of distinguishing CSW from the familiar cause of hyponatraemia SIADH; although the distinction can be challenging, it is absolutely essential as the treatments for the two conditions are so fundamentally different.

Pharmacological intervention also has a role. Fludrocortisone is recommended as a potential therapeutic option in CSW. In this reported case, the addition of fludrocortisone did appear to reduce the need for on-going boluses of hypertonic saline, likely through its mineralocorticoid potency which encourages sodium retention. Careful monitoring is still essential as overly aggressive fluid and sodium administration combined with mineralocorticoids can cause volume overload, leading to pulmonary oedema and hypertension. Tolvaptan a competitive $\mathrm{V} 2$ receptor antagonist is a recent novel treatment for hyponatraemia. However current opinion is that it should not be used in CSW as it is likely to exacerbate fluid depletion.

In conclusion, this report highlights the need for vigilance in encountering hyponatraemia in the neurosurgical patient, and the diagnostic challenge that is CSW. Furthermore, it clearly demonstrates the substantial volumes of hypertonic saline that can be required and how fludrocortisone can be used to mitigate this. It is noteworthy that although we have known about CSW for several decades several fundamental questions have yet to be answered, the most important of which are how cerebral injury leads to the release of excessive amounts of natriuretic peptides and why this occurs in only a small subset of cerebral injury types.

Declaration of interest

The authors declare that there is no conflict of interest that could be perceived as prejudicing the impartiality of the research reported.

\section{Funding}

This research did not receive any specific grant from any funding agency in the public, commercial or not-for-profit sector.

\section{Patient consent}

The patient has given full consent and is actually one of the authors (A W) who also happened to be a consultant endocrinologist!

\section{Author contribution statement}

P T helped manage the patient and drafted the manuscript. S D, A T, A S, $\mathrm{P} \mathrm{S}, \mathrm{H} \mathrm{B}$ and $\mathrm{P} \mathrm{K}$ helped collect the clinical information and also assisted in management of the patient pre and post discharge. M D and A R provided senior advice on managing the patient and also refined the manuscript. $\mathrm{CH}$ and S D were the senior clinicians responsible for the patient and helped refine the manuscript. A W was the patient and also a consultant endocrinologist who assisted with the refining of the manuscript.

\section{References}

1 Peters JP, Welt LG, Sims EA, Orloff J \& Needham J 1950 A saltwasting syndrome associated with cerebral disease. Transactions of the Association of American Physicians 63 57-64.

2 Berendes E, Walter M, Cullen P, Prien T, Van Aken H, Horsthemke J, Schulte M, von Wild K \& Scherer R 1997 Secretion of brain natriuretic 
Endocrinology

Diabetes \& Metabolism

CASE REPORTS

\section{P Taylor and others}

peptide in patients with aneurysmal subarachnoid haemorrhage. Lancet 349 245-249. (doi:10.1016/S0140-6736(96)08093-2)

3 Spasovski G, Vanholder R, Allolio B, Annane D, Ball S, Bichet D, Decaux G, Fenske W, Hoorn EJ, Ichai C, et al. 2014 Clinical practice guideline on diagnosis and treatment of hyponatraemia. European Journal of Endocrinology 170 G1-G47. (doi:10.1530/EJE-13-1020)

4 Tomida M, Muraki M, Uemura K \& Yamasaki K 1998 Plasma concentrations of brain natriuretic peptide in patients with

\section{CSW following TBI}

ID: 16-0142; April 2017

DOI: 10.1530/EDM-16-0142 subarachnoid hemorrhage. Stroke 29 1584-1587. (doi:10.1161/01. STR.29.8.1584)

5 Yee AH, Burns JD \& Wijdicks EF 2010 Cerebral salt wasting: pathophysiology, diagnosis, and treatment. Neurosurgery Clinics of North America 21 339-352. (doi:10.1016/j.nec.2009.10.011)

6 Betjes MG 2002 Hyponatremia in acute brain disease: the cerebral salt wasting syndrome. European Journal of Internal Medicine 13 9-14. (doi:10.1016/S0953-6205(01)00192-3)

Received in final form 26 February 2017

Accepted 2 March 2017 